\title{
Discussion Of Cavitation In FDAS Design
}

\author{
Wang Cheng ${ }^{1}$, Gu Lei ${ }^{1,2}$, Gu YIWei ${ }^{1}$, Ji Chen ${ }^{1}$ \\ ${ }^{1}$ Shanghai Marine Electronic Equipment Research Institute \\ ${ }^{2}$ Science and Technology on Underwater Acoustic Antagonizing Laboratory, Shanghai 201108, \\ China
}

15601751046@163.com

Keywords: cavitation, transducer, Low-frequency

\begin{abstract}
The Flexural Disc Assembled System is a promising transducer for the acoustic antagonizing device with low frequency and high power in limited size. The flexural disc Arranged axially and by exploiting the acoustic interaction between the benders, we can easily reduce the resonant frequency and improve the bandwidth. In our research, we find the receiving signal always distort which caused by cavitation at high power transmission.
\end{abstract}

\section{Introduction}

The transmitting capability of transducer is restricted by cavitation during high power transmission. The cavitation is defined as when the pressure inside the liquid is decreasing, the formation, growth and vanish of the liquid bubble or steam inside the liquid or at the liquid-solid interface. According to the dynamics concept, the cavitation can be catalogued into four different types and are shifting cavitation, fixed cavitation, vortex cavitation and oscillation cavitation respectively. Oscillation cavitation means a series of continuous high amplitude and high frequency pressure pulse which cause the growth or vanish of the bubble and steam. This series of pressure pulse is formed by the pressure wave where latent at the liquid and vibrate along the normal position. The pressure pulse can either squeeze or stretch the liquid, the cavitation phenomenon is observed as the pressure inside the liquid is less than the gasification pressure of the fluid.

\section{Cavitation Character In FDAS}

The underwater transducer generally produce diffusion wave. The transducer will induce cavitation since the power is increasing and it leads to the transducer can't transmit more power into water. It means the motion of fluid cannot follow with the motion of the transducer as the motion of the transducer is opposite toward fluid, and will cause the large stretch force at the liquid-solid interface. The cavitation will be emerged when the power of transducer is large enough to cause the liquid pressure placed at the liquid-solid interface is less than the gasification pressure.
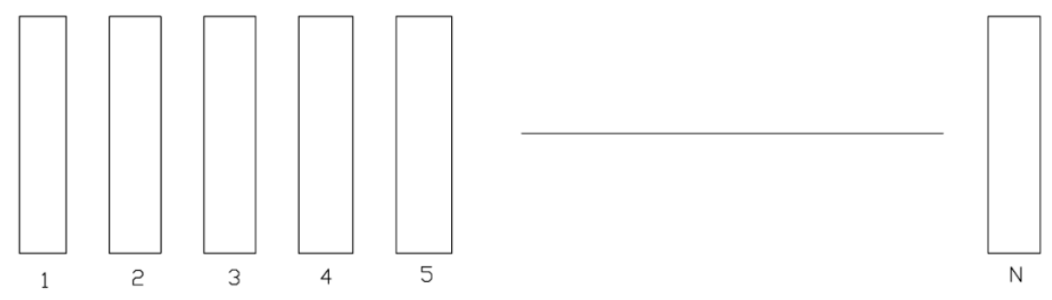

Fig.1 views of FDAS

The transducer are connected parallel to each other, the two adjacent transducers vibrate in the opposite direction. As the motion of transducer is opposite toward the fluid, the liquid-solid interface undergoes the stretch force created from the neighbor will also transfer to this interface. So the radiation plane undergoes more strong stretch force in the same power comparing with other type of transducer or projection systems and the cavitation will be more easily occurred. 
Another problem is the amplitude distribution at the flexural disk plane is uneven. Fig.7 shows the amplitude distribution of the plane and at the middle place of the disk presents the larger amplitude while the smaller amplitude occurs at the edge. The maximum value is ten times larger than the minimum value, while the interaction will further increase the ratio of maximum value and minimum value. Therefore, as the average power of transducer is far less than the cavitation power of the liquid, the cavitation may already occurred at larger amplitude place.

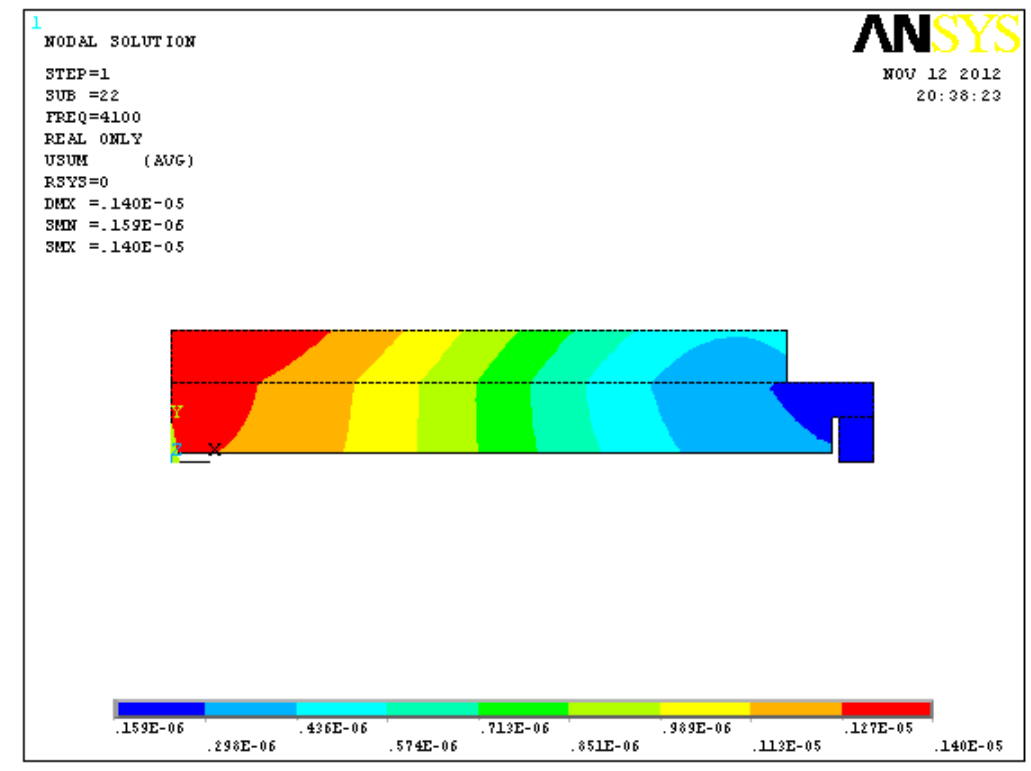

Fig.2 The amplitude distribution of flexural disk transducer

Additionally, the flatness of the transducer and the wettability of the sealing material will also affect the cavitation threshold. According to the report, the cavitation at the rough radiation plane will immediately occurred comparing to the smooth one at the same power projection. Besides, the cavitation also occurred quickly at the poorer wettability of the sealing material.

\section{Measure to improve}

For all the problems discussed above, we have developed a corresponding improvement measures and descript as follows:

1) Improve the flatness of the plane and the wettability of the sealing material. We grind the surface of the sealing mold in high precision and also invent a new mold manufacturing process without using the releasing agent which would adhesive at the surface of the transducer and cause to reduce the surface wettability.

2) In order to increase the amplitude at the center area, we increase the diameter as possible as we could and therefore reduce the largest amplitude at the radiation plane. We choose the most uniform distribution of the amplitude simulated by the Ansys.

\section{Experiment}

According to the research results discussed above, a new type of transducer has been developed based on the prototype built earlier. Fig. 3 gives the comparison between the new type bender element and the old one while fig.4 gives the system comparison respectively: 


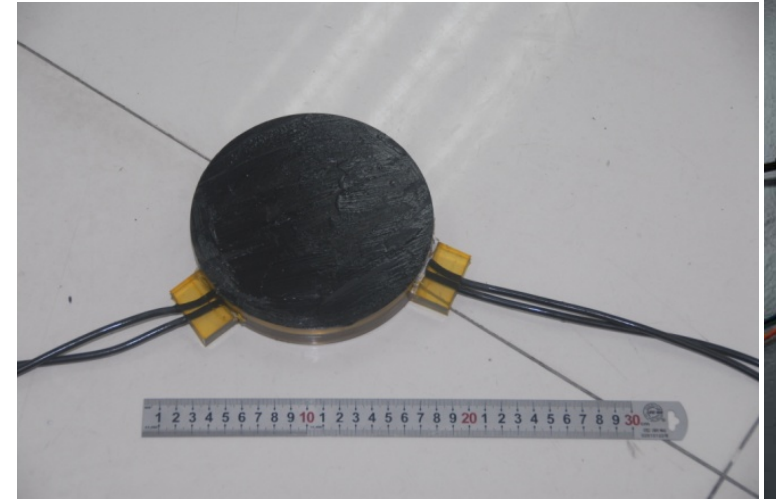

(a)

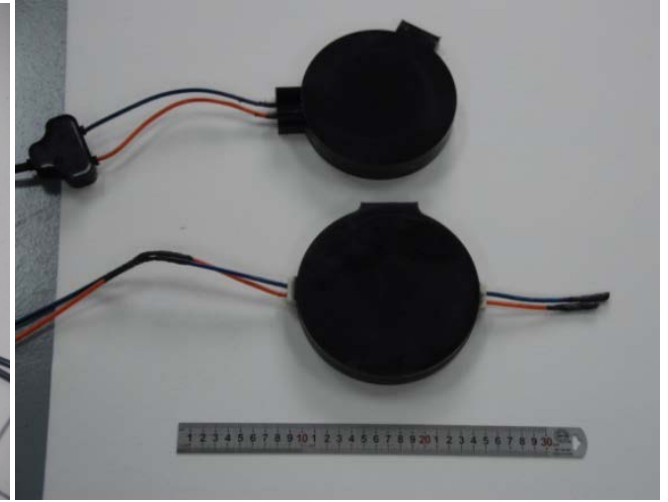

(b)

Fig.3 (a) old type bender, (b) new type bender

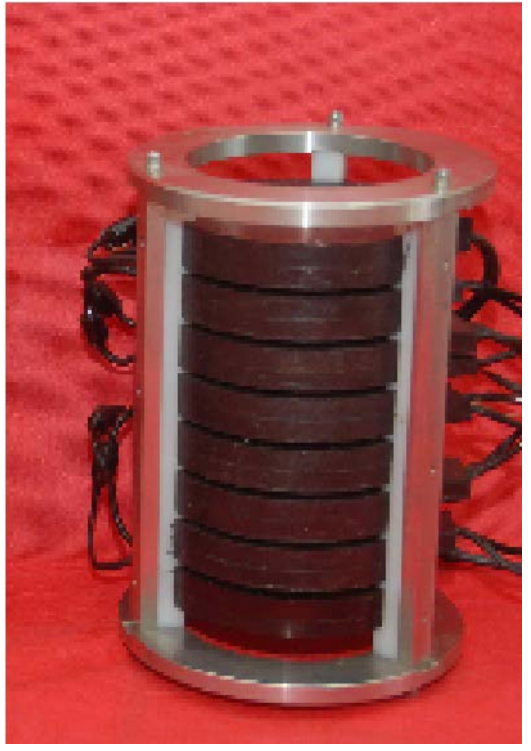

(a)

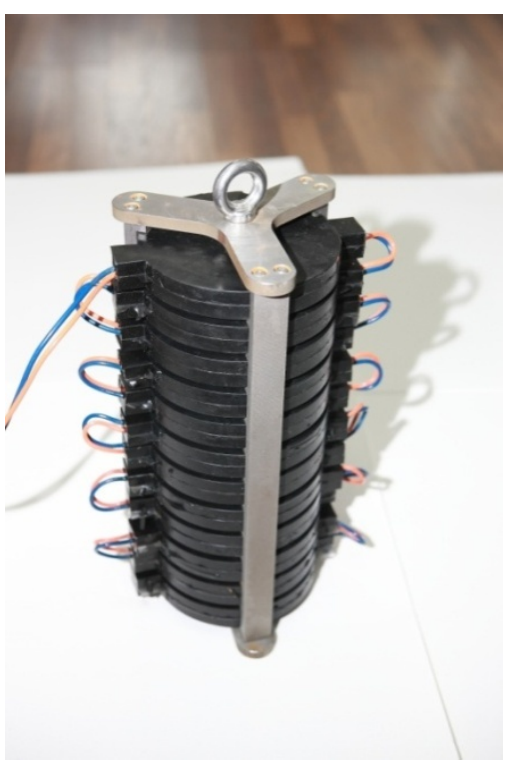

(b)

Fig.4 (a) old system, (b) new system

The testing data is given in Table 1:

Table 1: testing data

\begin{tabular}{|c|c|c|c|}
\hline $\begin{array}{c}\text { Text } \\
\text { frequency(Hz) }\end{array}$ & Depth(m) & $\begin{array}{c}\text { SL(dB) } \\
\text { Old type }\end{array}$ & $\begin{array}{c}\text { SL(dB) } \\
\text { New type }\end{array}$ \\
\hline 500 & 10 & 187 & 191 \\
\hline
\end{tabular}

\section{Summary}

The cavitation is the most important factor that affecting the source level of the transducer when it is placed at shallow water or the system is assembled in closely space. In this paper, by improving the manufacturing process for the flexural disk transducer and using the Ansys simulation to analysis the amplitude distribution, we can reduce the cavitation to some extent. But the only method to cancel the cavitation thoroughly is to put FDAS into deeper water.

\section{References}

[1] "The Towed Torpedo Emulator(TOTEM): A Tool for Testing and Training of Surface Ship Torpedo Defence Systems”,by M. Trevorrow,D.Smart,and S.De Belie,Sea Technology,March 2007,Vo1.48,No.3,pg41.

[2] “A Modular Projector System:Modeled Versus Measured Performance”,by J.Crawford, C.Purcell and B.Armstrong,presented at UDT Europe 2006(Winner of Best Paper Award), 
Hamburg, Germany,June 27-29,2006

[3] The research of new pattern of bender array by $\mathrm{Gu}$ Lei at Acoustics And Electronics Engineering, 2011,1,10-12 .

[4] P Yeatman, B.Armstrong, A Modular Projector System For Diverse Applications, UDT Europe 2005

[5] J.Crawford,C.Purcell and R. Fleming, “The Towed Torpedo Emulator(TOTEM): A Modular Projector System(MPS) Success Story”, presented at UDT Europe 2007,Naples,Italy,june5-7,2007 